\title{
Postharvest quality of Rambutan (Nephelium lappaceum L.) associated with packaging and storage conditions
}

\author{
Shetty J. Manjunath ${ }^{1}$ 凶, Geethalekshmy P.R. ${ }^{1}$, Mini C. ${ }^{1}$, Vijayaraghava kumar ${ }^{2}$ and Beena R. $^{3}$
}

\begin{abstract}
Limited information exists regarding the ideal packaging and storage conditions for optimum quality and storage life of rambutan. In this study, the physicochemical properties of rambutan fruits were monitored during storage. Fruit were packaged in Ventilated LDPE (150 gauge), Ventilated Polypropylene (PP) (100 gauge), Polystyrene tray with cling film wrap, Shrink wrapping and stored under room $\left(30 \pm 2^{\circ} \mathrm{C}\right.$ and $\left.\mathrm{RH} 80-85 \%\right)$ or refrigerated $\left(10 \pm 2^{\circ} \mathrm{C}\right.$ and $\left.\mathrm{RH} 90-95 \%\right)$ conditions were investigated for their potential to enhance the quality and extend the storage life of the fruit. Rambutan pre-packaged in ventilated polypropylene (PP) reduced weight loss. Refrigerated storage extended storage life of PP packed fruits compared to un-refrigerated fruits up to 14 days. Result of study revealed that Rambutan pre-packaging of rambutan in ventilated polypropylene (PP) reduced weight loss, while refrigerated storage extended storage life of $P P$ packed fruits compared to un-refrigerated fruits up to 14 days. These results provide important information regarding the storage behavior of rambutan fruits and indicate that packaging and storage conditions are effective for reducing moisture loss and extending storage life of fresh rambutan fruit.
\end{abstract}

Key words: Shelf life, Polypropylene, Polystyrene, Shrink wrap, Refrigeration

\section{Introduction}

Fruits and vegetables are highly perishable, as they continue their metabolic processes after harvest and these biological activities deteriorate the quality. The rate of respiration and multiplication of decay organisms is higher at high temperature (Desai et al., 1986). Produce must have less than $3 \%$ to $5 \%$ water loss to maintain its marketability. Wilting and shrivelling caused by water loss seriously damage the product appearance (Mitchell, 1991).After harvest rambutan fruits rarely stay marketable for more than 2-3 days under ambient conditions (Mendonza et al., 1972; Lam et al., 1987). The fruit pericarp, consisting of soft spines called spinterns which have more stomata than that of peel or main fruit axis (Pantastico et al., 1975). Extreme desiccation leads to browning of entire pericarp Physiological weight loss of a produce decreased horticultural perishables by reducing physical

Author's Address
${ }^{\mathbf{1} D e p a r t m e n t ~ o f ~ P o s t h a r v e s t ~ T e c h n o l o g y, ~ C o l l e g e ~ o f ~}$
Horticulture, Bengaluru, UHS (B), Karnataka
2Department of Agricultural Statistics, College of Agriculture,
Vellayani, KAU, Kerala
${ }^{\mathbf{3} D e p a r t m e n t ~ o f ~ P l a n t ~ P h y s i o l o g y, ~ C o l l e g e ~ o f ~ A g r i c u l t u r e, ~}$
Vellayani, KAU, Kerala
E-mail.: jshettymanju@gmail.com

Copyright by ASEA

All rights of reproduction in any form reserved injury during transit and handling (Gast, 1991). even if the pulp or aril is still acceptable or edible. Packaging minimizes the postharvest losses of with increase in thickness of plastic bags (Nainar et al., 1997). Suitable postharvest handling practices can enhance the shelf life by preserving its nutritional quality and there by extending the availability for domestic and distant market. Hence the study on packaging and storage of rambutan was done to extend the shelf life of rambutan with minimum nutritional loss.

\section{Material and methods}

Rambutan fruits of uniform maturity were harvested from the homesteads of Thiruvananthapuram districts and were prepackaged immediately after ozonization (2 ppm) treatment, with different packages (Plate 1.). Prepackaged fruits (150g each) were stored under two conditions in three replications. Macro ventilation of $2 \%$ was provided for LDPE and PP packages. Effectiveness of packaging materials was analyzed based on physiological and biochemical fruit quality parameters. Observations on PLW was recorded on alternate days whereas biochemical 
parameters were taken at an interval of two days till the end of shelf life. Effects of packaging treatments and storage conditions were statistically analyzed, found that treatments differed significantly. The data generated from experiments were statistically analyzed using Completely Randomized Design (CRD).

Pre-packaging treatments $\mathrm{P}_{1^{-}}$Ventilated LDPE (150 gauge)

$\mathrm{P}_{2}$ - Ventilated PP (100 gauge)

$\mathrm{P}_{3}$ - Polystyrene tray with cling film wrap

$\mathrm{P}_{4^{-}}$- Shrink wrapping

$\mathrm{P}_{5^{-}}$Control (without any pre-packaging)

Storage conditions

$\mathrm{S}_{1}$ - Room temperature $\left(30 \pm 2^{\circ} \mathrm{C}\right.$ and $\left.\mathrm{RH} 80-85 \%\right)$

$\mathrm{S}_{2^{-}}$Refrigerated condition $\left(10 \pm 2^{\circ} \mathrm{C}\right.$ and $\mathrm{RH} 90$ 95\%)

\section{Physiological loss in weight (PLW)}

For determining physiological loss in weight, sample was weighed accurately after the pretreatment and weight was taken daily till the end of shelf life and cumulative weight loss was calculated using the formula and expressed as percentage.

PLW $(\%) \frac{\text { Initial weight }- \text { Final weight }}{\text { Initial weight }} \times 100$

Total Soluble Solids:

Total Soluble Solids (TSS) of fruit pulp was recorded with digital refractrometer (Atago - 0 to $53{ }^{\circ} \mathrm{B}$ ) and expressed in ${ }^{0} \mathrm{~B}$.

pH: $\mathrm{pH}$ of the fruit pulp was measured by using pocket $\mathrm{pH}$ tester (HANNA instruments, pHep tester).

Titratable acidity: The titratable acidity was expressed in terms of per cent citric acid (Sadasivam and Manickam, 1992).

Vitamin C: Vitamin C content was estimated by 2,6- dichloro phenol indophenol (DCPIP) dye method (Ranganna, 1986) and expressed as mg/ $100 \mathrm{~g}$

Reducing sugar: The titrimetric method of Lane and Eynon (Ranganna, 1986) was adopted for the estimation of reducing sugar and expressed as per cent.

Non reducing sugar: The observations under total sugar and reducing sugar were used for calculating non-reducing sugar (Ranganna, 1986) and expressed as percent on fresh weight basis.
Total sugar: The total sugar content was expressed as per cent in terms of invert sugar (Ranganna, 1986).

Antioxidant activity: Total antioxidant activity of fruit pulp was determined using 2, 2- diphenyl-1picrylhydrazyl (DPPH) radical scavenging assay. The scavenging effect on DPPH free radical was measured (Sharma and Bhat, 2009).

Shelf life: $50 \%$ browning of spinterns is considered as the end of shelf life of stored rambutan fruits and expressed in days (O'Hare, 1995).

\section{Results and Discussion}

PLW of fruits stored at room temperature was $3.30 \%$ and $5.40 \%$ after 6 and 8 days of storage respectively whereas it was $2.19 \%$ and $3.10 \%$ respectively for fruits stored under refrigerated storage. Among the pre-packaging treatments, $\mathrm{P}_{2}$ (ventilated PP) had the lowest PLW of $2.52 \%$ after $8^{\text {th }}$ day of storage and the highest value was noticed in $\mathrm{P}_{5}$ (without pre-packaging) as $11.47 \%$. When the interaction effects were studied, treatment $\mathrm{P}_{2} \mathrm{~S}_{2}$ (ventilated $\mathrm{PP}+$ refrigerated storage) had the lowest PLW of $1.81 \%$ and the treatment $\mathrm{P}_{2} \mathrm{~S}_{1}$ (ventilated $\mathrm{PP}+$ room temperature) recorded lowest PLW of $3.23 \%$ for room temperature storage. The highest PLW of $4.03 \%$ was for $\mathrm{P}_{5} \mathrm{~S}_{2}$ (control + refrigerated storage) and $18.91 \%$ for $\mathrm{P}_{5} \mathrm{~S}_{1}$ (control + room temperature) after 8 days of storage. Fruits stored under room temperature were discarded due to spoilage after $8^{\text {th }}$ day of storage. The treatment $\mathrm{P}_{2} \mathrm{~S}_{2}$ (ventilated PP + refrigerated storage) recorded the lowest PLW of $2.83 \%$ and $3.38 \%$ after $12^{\text {th }}$ and $14^{\text {th }}$ day of storage respectively. The highest PLW was recorded in $\mathrm{P}_{5} \mathrm{~S}_{2}$ (control + refrigerated storage) after $12^{\text {th }}$ and $14^{\text {th }}$ days of storage as $7.04 \%$ and $9.91 \%$ respectively. Physiological loss in weight occurs after the harvest of fruits and is related to shelf life. Weight loss of rambutan is mainly due to respiration process and water loss from the fruit which not only reduce the quantity but also the sensory quality and marketability (Wills et al., 1989). When rambutans were pre-packaged in different packages, 100 gauge ventilated PP recorded the lowest weight loss after 8 days when stored at room temperature $(2.52 \%)$ and after 14 days of refrigerated storage $(3.38 \%)$ whereas unpacked fruits recorded highest PLW in both 
storage conditions. It might be due to the reduced transpiration and respiration rate of the fruits. Perforated bags reduced water and quality loss of rambutan fruits (Paine and Paine, (1992); O'Hare et al., 1994 and Widjanarko et al., 2000). Prepackaging has the potential to reduce moisture loss, restrict the entrance of oxygen, lower the rate of respiration and retard discoloration (Ahvenainen, 1996) and helps to increase shelf life by creating a modified atmosphere with an increase in concentration of carbon dioxide in the package (Assumi et al., 2009). Storage temperature plays an important role in PLW. Fruits stored under room temperature recorded the lowest PLW of $3.23 \%$ after 8 days after storage and also $3.28 \%$ under refrigerated condition after 14 days of storage (Fig. 1a and 1b). The lowest PLW was observed for fruits without any pre-packaging at both the storage conditions.

Rambutan fruits stored at room temperature recorded a TSS of $17.16^{\circ} \mathrm{B}$ after $8^{\text {th }}$ day of storage while it was $19.50{ }^{\circ} \mathrm{B}$ for refrigerated storage fruits. Among the pre-packaging treatments, after 8 days of storage, $\mathrm{P}_{2}$ (ventilated $\mathrm{PP}$ ) recorded the highest TSS of $18.76^{\circ} \mathrm{B}$ followed by $\mathrm{P}_{1}$ (ventilated LDPE) $\left(18.64{ }^{\circ} \mathrm{B}\right.$ ) while the lowest was in $\mathrm{P}_{5}$ (without prepackaging) $\left(17.13{ }^{\circ} \mathrm{B}\right)$. When the interaction effects were studied, The treatments $\mathrm{P}_{2} \mathrm{~S}_{2}$ (ventilated $\mathrm{PP}+$ room temperature) recorded highest TSS of (19.70 ${ }^{\circ} \mathrm{B}$ ) showing no difference with $\mathrm{P}_{1} \mathrm{~S}_{2}$ (ventilated LDPE + room temperature), $\mathrm{P}_{3} \mathrm{~S}_{2}$ (cling film wrap + room temperature), $\mathrm{P}_{4} \mathrm{~S}_{2}$ (shrink wrap + room temperature ranged between $19.53{ }^{\circ} \mathrm{B}$ and $19.63{ }^{\circ} \mathrm{B}$ and the lowest TSS was recorded in $\mathrm{P}_{5} \mathrm{~S}_{2}$ (control + refrigerated storage) as $19.06{ }^{\circ} \mathrm{B}$ after 8 days of storage at refrigerated storage. After $14^{\text {th }}$ day of storage, fruits showed no significant difference in TSS for the pre-packaging treatments $\left(\mathrm{P}_{1} \mathrm{~S}_{2}, \mathrm{P}_{2} \mathrm{~S}_{2}\right.$, $\mathrm{P}_{3} \mathrm{~S}_{2}, \mathrm{P}_{4} \mathrm{~S}_{2}$ ) under refrigerated storage and it ranged from $17.76{ }^{\circ} \mathrm{B}$ to $18.03{ }^{\circ} \mathrm{B}$ (Table 1). Pre-packaging as well as storage temperature influenced the biochemical parameters of rambutan during storage. TSS of all pre-packaged rambutan fruits decreased during the storage irrespective of the storage temperature. Loss of biochemical parameters was greater with increase in storage temperature and duration in fruits (Kays and Paull, (2004) and Onyango, (2010)). The decrease in TSS during storage might be due to rapid utilization of sugar and other metabolites for respiration. This was in agreement with Azene et al. (2014) who reported that TSS of papaya was unaltered by prepackaging and storage at $10^{\circ} \mathrm{C}$. Reduction of TSS observed during room temperature storage could be attributed to accelerate ripening due to high temperature. This could be due to slower conversion of soluble sugar (Tefera et al., 2007 and Getenit et al., 2008).

$\mathrm{pH}$ of rambutan fruits stored at room temperature recorded a pH of as 3.72 after 6 days of storage and 4.02 after 8 days of storage whereas it was 3.49 and 3.58 respectively under refrigerated storage (Table 2 ). Among the pre-packaging treatments, after $8^{\text {th }}$ day of storage where $\mathrm{P}_{2}$ (ventilated PP) had lowest $\mathrm{pH}$ of 3.73 which showed no difference with $\mathrm{P}_{4}$ (shrink wrap) and $\mathrm{P}_{1}$ (ventilated LDPE) while $\mathrm{P}_{5}$ (without pre-packaging) had maximum $\mathrm{pH}$ of 3.93. When interaction effects were studied, After $8^{\text {th }}$ day of storage, treatments $\mathrm{P}_{1} \mathrm{~S}_{2}, \mathrm{P}_{2} \mathrm{~S}_{2}, \mathrm{P}_{3} \mathrm{~S}_{2}$ and $\mathrm{P}_{4} \mathrm{~S}_{2}$ recorded no significant difference among themselves and highest $\mathrm{pH}$ of 3.66 was for $\mathrm{P}_{5} \mathrm{~S}_{2}$ (control + refrigerated storage). Similarly at room temperature storage, lowest $\mathrm{pH}$ was noticed in $\mathrm{P}_{1} \mathrm{~S}_{1}$, $\mathrm{P}_{2} \mathrm{~S}_{1}, \mathrm{P}_{3} \mathrm{~S}_{1}$ and $\mathrm{P}_{4} \mathrm{~S}_{1}$ showing no significant difference among themselves and the highest $\mathrm{pH}$ of 4.26 was for $\mathrm{P}_{5} \mathrm{~S}_{1}$ (control + room temperature). Rambutan fruits stored under room temperature were discarded due to spoilage after $8^{\text {th }}$ day of storage. After $14^{\text {th }}$ day of storage, ventilated PP + refrigerated storage $\left(\mathrm{P}_{2} \mathrm{~S}_{2}\right)$ recorded the lowest $\mathrm{pH}$ of 3.90 having no difference with $\mathrm{P}_{1} \mathrm{~S}_{2}$ (ventilated LDPE + refrigerated storage) and the highest $\mathrm{pH}$ was recorded in treatment $\mathrm{P}_{5} \mathrm{~S}_{2}$ (control + refrigerated storage) after $12^{\text {th }}$ and $14^{\text {th }}$ days of storage as 4.06 and 4.23 respectively.

Acidity of fruits stored at room temperature was $0.43 \%$ and $0.33 \%$ after 6 and 8 days of storage respectively while it was $0.55 \%$ and $0.52 \%$ respectively under refrigerated storage. Prepackaging treatments $\left(\mathrm{P}_{1}, \mathrm{P}_{2}, \mathrm{P}_{3}\right.$ and $\left.\mathrm{P}_{4}\right)$ showed no difference while minimum acidity was observed as $0.40 \%$ by $\mathrm{P}_{5}$ (without pre-packaging). When the interaction effects were studied, after $8^{\text {th }}$ day of storage, treatments $\mathrm{P}_{2} \mathrm{~S}_{2}$ (ventilated $\mathrm{PP}+$ refrigerated storage) $(0.54 \%), \mathrm{P}_{1} \mathrm{~S}_{2}$ (ventilated LDPE+ refrigerated storage) $(0.54 \%), \mathrm{P}_{3} \mathrm{~S}_{2}$ (cling film wrap+ refrigerated storage) $(0.52 \%)$ and $\mathrm{P}_{4} \mathrm{~S}_{2}$ (shrink wrap + refrigerated storage) $(0.52 \%)$ did not differ significantly. The treatment $\mathrm{P}_{5} \mathrm{~S}_{2}$ (ventilated $\mathrm{PP}+$ room temperature) recorded lowest acidity of 
$0.50 \%$ at refrigerated storage. Pre-packaging treatments $\left(\mathrm{P}_{1} \mathrm{~S}_{1}, \mathrm{P}_{2} \mathrm{~S}_{1}, \mathrm{P}_{3} \mathrm{~S}_{1}\right.$ and $\left.\mathrm{P}_{4} \mathrm{~S}_{1}\right)$ showed no significant difference under room temperature and the lowest acidity was recorded for $\mathrm{P}_{5} \mathrm{~S}_{1}$ (control + room temperature) $(0.29 \%)$ after $8^{\text {th }}$ day of storage. Fruits stored under room temperature were discarded due to spoilage after $8^{\text {th }}$ day of storage. After $14^{\text {th }}$ day of storage, ventilated $\mathrm{PP}+$ refrigerated storage $\left(\mathrm{P}_{2} \mathrm{~S}_{2}\right)$ recorded the highest acidity of $0.44 \%$ showed no difference with $\mathrm{P}_{1} \mathrm{~S}_{2}$ (ventilated LDPE + refrigerated storage) $(0.39 \%)$. The lowest acidity was recorded in treatment $\mathrm{P}_{5} \mathrm{~S}_{2}$ (control + refrigerated storage) after $12^{\text {th }}$ and $14^{\text {th }}$ days of storage as $0.32 \%$ and $0.25 \%$ respectively (Table 3). $\mathrm{pH}$ was found to be increasing during storage of rambutan fruits whereas acidity was decreasing in all the treatments. This might be due to the reason that organic acids present in fruits were utilized during respiration process as substrates. In case of storage temperature, the fruits stored under refrigerated condition recorded highest acidity $(0.50 \%)$ and lowest $\mathrm{pH}$ (3.66). $\mathrm{pH}$ and acidity showed no difference among pre-packaging treatments after $8^{\text {th }}$ day of storage. When interaction effect was considered, pre-packaged rambutan stored under room temperature recorded no difference for $\mathrm{pH}$ and acidity after 8 days of storage whereas ventilated PP recorded lowest $\mathrm{pH}$ (3.90) and highest acidity $(0.44 \%)$ was noticed for rambutan fruits under refrigerated condition after 14 days of storage. Depletion of organic acids stored at room temperature can be attributed to the faster ripening rate of fruits and respiration (Wills et al., 1989). Pantastico (1995) reported that increase in $\mathrm{pH}$ during storage is due to conversion of organic acids in to sugar.

Fruits stored at room temperature recorded vitamin $\mathrm{C}$ of $23.49 \mathrm{mg} / 100 \mathrm{~g}$ after 6 days of storage and $20.40 \mathrm{mg} / 100 \mathrm{~g}$ under room temperature after 8 days of storage whereas it was $26.29 \mathrm{mg} / 100 \mathrm{~g}$ and 25.27 $\mathrm{mg} / 100 \mathrm{~g}$ respectively under refrigerated storage. After $8^{\text {th }}$ day of storage, $\mathrm{P}_{2}$ (ventilated PP) had highest vitamin $C$ content of $23.87 \mathrm{mg} / 100 \mathrm{~g}$ which was non-significant with shrink wrapping $\left(\mathrm{P}_{4}\right)$ $(23.74 \mathrm{mg} / 100 \mathrm{~g})$ and ventilated $\operatorname{LDPE}\left(\mathrm{P}_{1}\right)$. Lowest vitamin $\mathrm{C}$ was noticed in $\mathrm{P}_{5}$ (without prepackaging) as $23.06 \mathrm{mg} / 100 \mathrm{~g}$ and $20.85 \mathrm{mg} / 100 \mathrm{~g}$ after $6^{\text {th }}$ and $8^{\text {th }}$ day of storage respectively. When the interaction effects were studied, after $8^{\text {th }}$ day of storage $\mathrm{P}_{2} \mathrm{~S}_{2}$ (ventilated $\mathrm{PP}+$ refrigerated storage) recorded $26.35 \mathrm{mg} / 100 \mathrm{~g}$ with no significant difference with $\mathrm{P}_{4} \mathrm{~S}_{2}$ (shrink wrapping + refrigerated storage). The treatment $\mathrm{P}_{2} \mathrm{~S}_{1}$ (ventilated $\mathrm{PP}+$ room temperature) recorded a vitamin $\mathrm{C}$ content of $21.43 \mathrm{mg} / 100 \mathrm{~g}$ which had no difference with $\mathrm{P}_{4} \mathrm{~S}_{1}$ (shrink wrapping + room temperature) and $\mathrm{P}_{1} \mathrm{~S}_{1}$ (ventilated $\mathrm{LDPE}+$ room temperature) under room temperature. The lowest vitamin $\mathrm{C}$ content of $23.62 \mathrm{mg} / 100 \mathrm{~g}$ was for $\mathrm{P}_{5} \mathrm{~S}_{2}$ (control + refrigerated storage) and $18.07 \mathrm{mg} / 100 \mathrm{~g}$ for $\mathrm{P}_{5} \mathrm{~S}_{1}$ (control + room temperature) after $8^{\text {th }}$ day of storage. Fruits stored under room temperature were discarded due to spoilage after $8^{\text {th }}$ day of storage. After $14^{\text {th }}$ day of storage, ventilated $\mathrm{PP}+$ refrigerated storage $\left(\mathrm{P}_{2} \mathrm{~S}_{2}\right)$ recorded the highest vitamin $\mathrm{C}$ content of $22.27 \mathrm{mg} / 100 \mathrm{~g}$ showed no significant difference with $\mathrm{P}_{4} \mathrm{~S}_{2}$ (shrink wrapping+ refrigerated storage) $21.75 \mathrm{mg} / 100 \mathrm{~g}$.

The lowest vitamin $\mathrm{C}$ content was recorded in treatment $\mathrm{P}_{5} \mathrm{~S}_{2}$ (control + refrigerated storage) after $12^{\text {th }}$ and $14^{\text {th }}$ days of storage as $18.44 \mathrm{mg} / 100 \mathrm{~g}$ and $16.16 \mathrm{mg} / 100 \mathrm{~g}$ respectively. Rambutan prepackaged in ventilated PP showed highest vitamin $\mathrm{C}$ of $23.87 \mathrm{mg} / 100 \mathrm{~g}$ and after 8 days of storage at room temperature and $(22.27 \mathrm{mg} / 100 \mathrm{~g})$ at the end of shelf life at 14 days of refrigerated storage (Fig. $2 \mathrm{a}$ and $2 \mathrm{~b}$ ). Vitamin $\mathrm{C}$ is sensitive to oxygen as it is a reducing agent and creation of modified atmosphere around the fruits through packaging prevents reduction of vitamin C. During storage it was found that refrigerated storage retained more vitamin C $(25.27 \mathrm{mg} / 100 \mathrm{~g})$ than at room temperature but a slight decrease was observed from initial content which may be due to lower rate of conversion of ascorbic acid to dehydroascorbic acid at low temperature and conversion is hindered by reduced metabolic and enzymatic activity. Similar results were presented by Singh and Rao (2005) where papaya fruits packed with polypropylene had consistently high vitamin $\mathrm{C}$ than other pre-packaging treatments after 15 days of storage at low temperature. Reducing sugar of fruits stored at room temperature was $5.68 \%$ after 6 days of storage and $4.96 \%$ after 8 days of storage while it was $6.64 \%$ and $6.25 \%$ for fruits after $6^{\text {th }}$ and $8^{\text {th }}$ day respectively for fruits stored under refrigerated storage. After 8 days of storage, $\mathrm{P}_{1}$ (ventilated LDPE), $\mathrm{P}_{2}$ (ventilated PP), $\mathrm{P}_{3}$ (cling film wrap) and $\mathrm{P}_{4}$ (shrink wrap) showed no significant difference while the $\mathrm{P}_{5}$ (without pre-packaging) had lowest 
Postharvest quality of Rambutan (Nephelium lappaceum $\mathbf{L}$.)

Table 1. Effect of pre-packaging on TSS $\left({ }^{\circ} \mathrm{B}\right)$ of rambutan fruits under different storage conditions

\begin{tabular}{|c|c|c|c|c|c|c|c|c|c|c|c|c|c|c|c|c|c|c|}
\hline \multirow{4}{*}{$\begin{array}{l}\text { Pre- } \\
\text { packag } \\
\text { ing } \\
\text { Treat } \\
\text { ments }\end{array}$} & \multicolumn{18}{|c|}{ Total Soluble Solids $\left({ }^{\circ} \mathbf{B}\right)$} \\
\hline & \multicolumn{18}{|c|}{ Days after storage } \\
\hline & \multicolumn{3}{|c|}{ At storage } & \multicolumn{3}{|c|}{2} & \multicolumn{3}{|c|}{4} & \multicolumn{3}{|c|}{6} & \multicolumn{3}{|c|}{8} & \multirow{2}{*}{$\begin{array}{c}10 \\
\text { (*) } \mathrm{S}_{2}\end{array}$} & \multirow{2}{*}{$\begin{array}{l}12 \\
\mathrm{~S}_{2}\end{array}$} & \multirow{2}{*}{$\begin{array}{r}14 \\
S_{2}\end{array}$} \\
\hline & $\mathrm{S}_{1}$ & $\mathrm{~S}_{2}$ & $\begin{array}{l}\text { Mean } \\
\text { (P) }\end{array}$ & $\mathrm{S}_{1}$ & $\mathrm{~S}_{2}$ & $\begin{array}{l}\text { Mean } \\
\text { (P) }\end{array}$ & $\mathrm{S}_{1}$ & $\mathrm{~S}_{2}$ & $\begin{array}{l}\text { Mean } \\
\text { (P) }\end{array}$ & $\mathrm{S}_{1}$ & $\mathrm{~S}_{2}$ & $\begin{array}{l}\text { Mean } \\
\text { (P) }\end{array}$ & $S_{1}$ & $\mathrm{~S}_{2}$ & $\begin{array}{l}\text { Mean } \\
\text { (P) }\end{array}$ & & & \\
\hline $\mathrm{P}_{1}$ & 20.26 & 20.26 & 20.26 & 20.03 & 20.23 & 20.15 & 19.10 & 20.03 & 19.58 & 18.33 & 19.63 & 18.98 & 17.66 & 19.63 & 18.64 & 19.16 & 18.33 & 17.96 \\
\hline $\mathrm{P}_{2}$ & 20.26 & 20.26 & 20.26 & 20.06 & 20.26 & 20.15 & 19.13 & 20.16 & 19.63 & 18.36 & 19.70 & 19.03 & 17.83 & 19.70 & 18.76 & 19.23 & 18.46 & 18.03 \\
\hline $\mathrm{P}_{3}$ & 20.20 & 20.20 & 20.20 & 20.00 & 20.20 & 20.10 & 18.96 & 20.03 & 19.50 & 18.23 & 19.53 & 18.88 & 17.46 & 19.53 & 18.50 & 19.13 & 18.26 & 17.76 \\
\hline $\mathrm{P}_{4}$ & 20.26 & 20.26 & 20.26 & 20.00 & 20.23 & 20.03 & 18.86 & 20.06 & 19.46 & 18.30 & 19.60 & 18.95 & 17.66 & 19.60 & 18.63 & 19.13 & 18.26 & 17.90 \\
\hline $\mathrm{P}_{5}$ & 20.26 & 20.26 & 20.26 & 19.90 & 20.10 & 20.00 & 17.43 & 19.90 & 18.66 & 16.13 & 19.06 & 17.60 & 15.20 & 19.06 & 17.13 & 17.63 & 16.73 & 15.76 \\
\hline $\begin{array}{l}\text { Mean } \\
\text { (S) }\end{array}$ & 20.26 & 20.26 & & 19.88 & 20.20 & & 18.70 & 20.04 & & 17.87 & 19.50 & & 17.16 & 19.50 & & 18.85 & 18.00 & 17.48 \\
\hline $\begin{array}{l}\text { CD } \\
(0.05)\end{array}$ & $\begin{array}{l}\text { P- NS } \\
\text { S-NS } \\
\text { PxS- NS }\end{array}$ & & & $\begin{array}{l}\text { P- } 0.094 \\
\text { S- } 0.059 \\
\text { PxS-NS }\end{array}$ & & & $\begin{array}{l}\text { P- } 0.150 \\
\text { S- } 0.09 \\
\text { PxS- } 0 .\end{array}$ & & & $\begin{array}{l}\text { P- } 0.147 \\
\text { S- } 0.09 \\
\text { PxS- } 0.2\end{array}$ & & & $\begin{array}{l}\text { P- } 0.17 \\
\text { S- } 0.11 \\
\text { PxS- } 0 .\end{array}$ & & & 0.196 & 0.470 & 0.493 \\
\hline
\end{tabular}

* - Rambutan fruits stored under room temperature $\left(S_{1}\right)$ were discarded due to spoilage after $8^{\text {th }}$ day of storage

$\mathrm{S}_{1}$ - Room temperature

$\mathrm{S}_{2}$ - Refrigerated storage

$\mathrm{P}_{1^{-}}$Ventilated LDPE, $\mathrm{P}_{2}$-Ventilated $\mathrm{PP}, \mathrm{P}_{3^{-}}$Polystyrene tray with cling film wrap, $\mathrm{P}_{4}-$ Shrink wrap, $\mathrm{P}_{5^{-}}$Control 
Shetty et al.

Table 2. Effect of pre-packaging on $\mathrm{pH}$ of rambutan fruits under different storage conditions

\begin{tabular}{|c|c|c|c|c|c|c|c|c|c|c|c|c|c|c|c|c|c|c|}
\hline \multirow{4}{*}{$\begin{array}{l}\text { Pre- } \\
\text { packagi } \\
\text { ng } \\
\text { Treatme } \\
\text { nts }\end{array}$} & \multicolumn{18}{|c|}{ pH } \\
\hline & \multicolumn{18}{|c|}{ Days after storage } \\
\hline & \multicolumn{3}{|c|}{ At storage } & \multicolumn{3}{|c|}{2} & \multicolumn{3}{|c|}{4} & \multicolumn{3}{|c|}{6} & \multicolumn{3}{|c|}{8} & \multirow{2}{*}{$\frac{10}{(*) S_{2}}$} & \multirow{2}{*}{$\begin{aligned} 12 \\
S_{2}\end{aligned}$} & \multirow{2}{*}{$\begin{array}{r}14 \\
S_{2}\end{array}$} \\
\hline & $\mathrm{S}_{1}$ & $\mathrm{~S}_{2}$ & $\begin{array}{l}\text { Mean } \\
\text { (P) }\end{array}$ & $\mathrm{S}_{1}$ & $\mathrm{~S}_{2}$ & $\begin{array}{l}\text { Mea } \\
n \\
(\mathrm{P}) \\
\end{array}$ & $\mathrm{S}_{1}$ & $\mathrm{~S}_{2}$ & $\begin{array}{l}\text { Mea } \\
\mathrm{n} \\
(\mathrm{P}) \\
\end{array}$ & $\mathrm{S}_{1}$ & $\mathrm{~S}_{2}$ & $\begin{array}{l}\text { Mea } \\
\mathrm{n} \\
(\mathrm{P}) \\
\end{array}$ & $\mathrm{S}_{1}$ & $\mathrm{~S}_{2}$ & $\begin{array}{l}\text { Mea } \\
\text { n (P) }\end{array}$ & & & \\
\hline $\mathrm{P}_{1}$ & 3.43 & 3.43 & 3.43 & 3.43 & 3.43 & 3.43 & 3.46 & 3.43 & 3.45 & 3.66 & 3.46 & 3.56 & 4.00 & 3.60 & 3.76 & 3.76 & 3.83 & 3.93 \\
\hline $\mathrm{P}_{2}$ & 3.43 & 3.43 & 3.43 & 3.33 & 3.33 & 3.33 & 3.33 & 3.33 & 3.33 & 3.63 & 3.43 & 3.53 & 3.93 & 3.53 & 3.73 & 3.60 & 3.76 & 3.90 \\
\hline $\mathrm{P}_{3}$ & 3.46 & 3.46 & 3.46 & 3.50 & 3.43 & 3.46 & 3.50 & 3.43 & 3.46 & 3.76 & 3.53 & 3.65 & 3.96 & 3.60 & 3.78 & 3.83 & 3.96 & 4.13 \\
\hline $\mathrm{P}_{4}$ & 3.43 & 3.43 & 3.43 & 3.46 & 3.43 & 3.45 & 3.53 & 3.43 & 3.48 & 3.76 & 3.50 & 3.63 & 3.96 & 3.53 & 3.80 & 3.80 & 3.93 & 4.06 \\
\hline $\mathrm{P}_{5}$ & 3.43 & 3.43 & 3.43 & 3.50 & 3.46 & 3.48 & 3.53 & 3.46 & 3.50 & 3.76 & 3.53 & 3.65 & 4.26 & 3.66 & 3.93 & 3.86 & 4.06 & 4.23 \\
\hline $\begin{array}{l}\text { Mean } \\
\text { (S) }\end{array}$ & 3.44 & 3.44 & & 3.44 & 3.42 & & 3.48 & 3.42 & & 3.72 & 3.49 & & 4.02 & 3.58 & & 3.77 & 3.90 & 4.05 \\
\hline $\begin{array}{l}C D \\
(0.05)\end{array}$ & $\begin{array}{l}\text { P- NS } \\
\text { S- NS } \\
\text { PxS- }\end{array}$ & & & $\begin{array}{l}\text { P-0.1 } \\
\text { S-0.0 } \\
\text { PxS-C }\end{array}$ & & & $\begin{array}{l}\text { P-0.1 } \\
\text { S-0.0 } \\
\text { PxS-C }\end{array}$ & & & $\begin{array}{l}\text { P- } 0.0 \\
\text { S- } 0.0 \\
\text { PxS-C }\end{array}$ & $\begin{array}{l}4 \\
120\end{array}$ & & $\begin{array}{l}\text { P-0.0 } \\
\text { S-0.0 } \\
\text { PxS- }\end{array}$ & $\begin{array}{l}9 \\
26 \\
125\end{array}$ & & 0.110 & 0.100 & 0.145 \\
\hline
\end{tabular}

$\mathrm{S}_{1}$ - Room temperature $\quad \mathrm{S}_{2}$ - Refrigerated storage

* - Rambutan fruits stored under room temperature $\left(S_{1}\right)$ were discarded due to spoilage after $8^{\text {th }}$ day of storage

$\mathrm{P}_{1^{-}}$- Ventilated LDPE, $\mathrm{P}_{2}$-Ventilated $\mathrm{PP}, \mathrm{P}_{3^{-}}$Polystyrene tray with cling film wrap, $\mathrm{P}_{4}-$ Shrink wrap, $\mathrm{P}_{5^{-}}$Control 
Postharvest quality of Rambutan (Nephelium lappaceum $\mathbf{L}$. )

Table 3. Effect of pre-packaging on acidity (\%) of rambutan fruits under different storage conditions

\begin{tabular}{|c|c|c|c|c|c|c|c|c|c|c|c|c|c|c|c|c|c|c|}
\hline \multirow{4}{*}{$\begin{array}{l}\text { Pre- } \\
\text { packagi } \\
\text { ng } \\
\text { Treatme } \\
\text { nts }\end{array}$} & \multicolumn{18}{|c|}{ Acidity (\%) } \\
\hline & \multicolumn{18}{|c|}{ Days after storage } \\
\hline & \multicolumn{3}{|c|}{ At storage } & \multicolumn{3}{|c|}{2} & \multicolumn{3}{|c|}{4} & \multicolumn{3}{|c|}{6} & \multicolumn{3}{|c|}{8} & \multirow{2}{*}{$\begin{array}{cc} & 10 \\
(*) S_{2}\end{array}$} & \multirow{2}{*}{$\begin{array}{r}12 \\
S_{2}\end{array}$} & \multirow{2}{*}{ 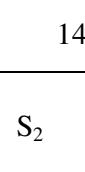 } \\
\hline & $\mathrm{S}_{1}$ & $\mathrm{~S}_{2}$ & $\begin{array}{l}\text { Mean } \\
(\mathrm{P})\end{array}$ & $\mathrm{S}_{1}$ & $\mathrm{~S}_{2}$ & $\begin{array}{l}\text { Mea } \\
\mathrm{n}(\mathrm{P})\end{array}$ & $\mathrm{S}_{1}$ & $\mathrm{~S}_{2}$ & $\begin{array}{l}\text { Mea } \\
\text { n (P) }\end{array}$ & $\mathrm{S}_{1}$ & $\mathrm{~S}_{2}$ & $\begin{array}{l}\text { Mea } \\
\text { n (P) }\end{array}$ & $\mathrm{S}_{1}$ & $\mathrm{~S}_{2}$ & $\begin{array}{l}\text { Mean } \\
\text { (P) }\end{array}$ & & & \\
\hline $\mathrm{P}_{1}$ & 0.58 & 0.58 & 0.58 & 0.57 & 0.59 & 0.58 & 0.54 & 0.58 & 0.56 & 0.44 & 0.55 & 0.49 & 0.34 & 0.54 & 0.44 & 0.49 & 0.44 & 0.39 \\
\hline $\mathrm{P}_{2}$ & 0.59 & 0.59 & 0.59 & 0.58 & 0.59 & 0.59 & 0.54 & 0.59 & 0.56 & 0.45 & 0.56 & 0.50 & 0.35 & 0.54 & 0.44 & 0.58 & 0.50 & 0.44 \\
\hline $\mathrm{P}_{3}$ & 0.59 & 0.59 & 0.59 & 0.57 & 0.58 & 0.57 & 0.52 & 0.58 & 0.55 & 0.42 & 0.55 & 0.48 & 0.33 & 0.52 & 0.42 & 0.47 & 0.41 & 0.35 \\
\hline $\mathrm{P}_{4}$ & 0.58 & 0.58 & 0.58 & 0.57 & 0.58 & 0.58 & 0.52 & 0.58 & 0.55 & 0.42 & 0.55 & 0.48 & 0.32 & 0.52 & 0.42 & 0.47 & 0.41 & 0.35 \\
\hline $\mathrm{P}_{5}$ & 0.59 & 0.59 & 0.59 & 0.58 & 0.58 & 0.58 & 0.50 & 0.58 & 0.54 & 0.39 & 0.55 & 0.47 & 0.29 & 0.50 & 0.40 & 0.44 & 0.32 & 0.25 \\
\hline $\begin{array}{l}\text { Mean } \\
\text { (S) }\end{array}$ & 0.58 & 0.58 & & 0.57 & 0.58 & & 0.52 & 0.58 & & 0.43 & 0.55 & & 0.33 & 0.52 & & 0.49 & 0.41 & 0.35 \\
\hline $\begin{array}{l}\text { CD } \\
(0.05)\end{array}$ & $\begin{array}{l}\text { P- N } \\
\text { S- N } \\
\text { PxS- }\end{array}$ & & & $\begin{array}{l}\text { P-N } \\
\text { S-N } \\
\text { PxS }\end{array}$ & & & $\begin{array}{l}\text { P- } 0.0 \\
\text { S- } 0.0 \\
\text { PxS- }\end{array}$ & & & $\begin{array}{l}\text { P- } 0.0 \\
\text { S- } 0.0 \\
\text { PxS- }\end{array}$ & & & $\begin{array}{l}\text { P- } 0.0 \\
\text { S- } 0.0 \\
\text { PxS- }\end{array}$ & & & 0.078 & 0.044 & 0.056 \\
\hline
\end{tabular}

$\mathrm{S}_{1}$ - Room temperature $\quad \mathrm{S}_{2}$ - Refrigerated storage

* - Rambutan fruits stored under room temperature $\left(\mathrm{S}_{1}\right)$ were discarded due to spoilage after $8^{\text {th }}$ day of storage

$\mathrm{P}_{1^{-}}$Ventilated LDPE, $\mathrm{P}_{2}$-Ventilated PP, $\mathrm{P}_{3^{-}}$Polystyrene tray with cling film wrap, $\mathrm{P}_{4}-$ Shrink wrap, $\mathrm{P}_{5^{-}}$Control 
Shetty et al.

Table 4. Effect of pre-packaging on total sugar (\%) of rambutan fruits under different storage conditions

\begin{tabular}{|c|c|c|c|c|c|c|c|c|c|c|c|c|c|c|c|c|c|c|}
\hline \multirow{4}{*}{$\begin{array}{l}\text { Pre } \\
- \\
\text { pac } \\
\text { kag } \\
\text { ing } \\
\text { Tre } \\
\text { atm } \\
\text { ent } \\
\text { s } \\
\end{array}$} & \multicolumn{18}{|c|}{ Total sugar (\%) } \\
\hline & \multicolumn{18}{|c|}{ Days after storage } \\
\hline & \multicolumn{3}{|c|}{ At storage } & \multicolumn{3}{|c|}{2} & \multicolumn{3}{|c|}{4} & \multicolumn{3}{|c|}{6} & \multicolumn{3}{|c|}{8} & \multirow{2}{*}{$\begin{array}{l}10 \\
(*) \\
S_{2}\end{array}$} & \multirow{2}{*}{$\begin{array}{r}12 \\
S_{2}\end{array}$} & \multirow{2}{*}{$\begin{array}{r}14 \\
S_{2}\end{array}$} \\
\hline & $\mathrm{S}_{1}$ & $\mathrm{~S}_{2}$ & $\begin{array}{l}\text { Mean } \\
(\mathrm{P})\end{array}$ & $\mathrm{S}_{1}$ & $\mathrm{~S}_{2}$ & $\begin{array}{l}\text { Mean } \\
(\mathrm{P})\end{array}$ & $\mathrm{S}_{1}$ & $\mathrm{~S}_{2}$ & $\begin{array}{l}\text { Mean } \\
(\mathrm{P})\end{array}$ & $\mathrm{S}_{1}$ & $\mathrm{~S}_{2}$ & $\begin{array}{l}\text { Mean } \\
\text { (P) }\end{array}$ & $\mathrm{S}_{1}$ & $\mathrm{~S}_{2}$ & $\begin{array}{l}\text { Mean } \\
\text { (P) }\end{array}$ & & & \\
\hline $\mathrm{P}_{1}$ & 19.27 & 19.27 & 19.27 & 18.38 & 18.56 & 18.47 & 18.01 & 18.49 & 18.25 & 16.44 & 17.23 & 16.84 & 15.17 & 16.98 & 16.17 & 16.28 & 15.11 & 14.13 \\
\hline $\mathrm{P}_{2}$ & 19.14 & 19.14 & 19.14 & 18.90 & 19.08 & 18.96 & 18.78 & 18.94 & 18.86 & 16.88 & 17.49 & 17.18 & 15.70 & 17.33 & 16.51 & 18.54 & 17.62 & 15.45 \\
\hline $\mathrm{P}_{3}$ & 19.21 & 19.21 & 19.21 & 18.40 & 18.94 & 18.74 & 18.32 & 18.58 & 18.45 & 16.65 & 17.32 & 16.99 & 15.10 & 16.81 & 16.05 & 16.25 & 15.03 & 14.11 \\
\hline $\mathrm{P}_{4}$ & 19.27 & 19.27 & 19.27 & 18.54 & 19.02 & 18.81 & 18.67 & 18.88 & 18.77 & 16.80 & 17.47 & 17.13 & 15.51 & 17.11 & 16.31 & 16.43 & 15.37 & 14.22 \\
\hline $\mathrm{P}_{5}$ & 19.21 & 19.21 & 19.21 & 18.12 & 18.60 & 18.36 & 17.88 & 18.30 & 17.94 & 14.65 & 16.60 & 15.62 & 12.47 & 15.58 & 14.02 & 14.22 & 13.19 & 11.74 \\
\hline $\begin{array}{l}\mathrm{Me} \\
\text { an } \\
(\mathrm{S})\end{array}$ & 19.22 & 19.23 & & 18.48 & 18.84 & & 18.33 & 18.45 & & 16.29 & 17.22 & & 14.87 & 16.76 & & 16.34 & 15.26 & 13.93 \\
\hline $\begin{array}{l}\text { CD } \\
(0.0 \\
5)\end{array}$ & $\begin{array}{l}\text { P- NS } \\
\text { S- NS } \\
\text { PxS- N }\end{array}$ & & & $\begin{array}{l}\text { P-0.46 } \\
\text { S-0.29 } \\
\text { PxS-0 }\end{array}$ & & & $\begin{array}{l}\text { P-0.49 } \\
\text { S-0.31 } \\
\text { PxS-0 }\end{array}$ & & & $\begin{array}{l}\text { P-0.61 } \\
\text { S-0.39 } \\
\text { PxS-0 }\end{array}$ & & & $\begin{array}{l}\text { P-0.58 } \\
\text { S-0.37 } \\
\text { PxS-0. }\end{array}$ & & & 0.588 & 0.613 & 0.744 \\
\hline
\end{tabular}

$\mathrm{S}_{1}$ - Room temperature $\quad \mathrm{S}_{2}$ - Refrigerated storage

* - Rambutan fruits stored under room temperature $\left(S_{1}\right)$ were discarded due to spoilage after $8^{\text {th }}$ day of storage

$\mathrm{P}_{1}$ - Ventilated LDPE, $\mathrm{P}_{2}$-Ventilated $\mathrm{PP}, \mathrm{P}_{3}$ - Polystyrene tray with cling film wrap, $\mathrm{P}_{4}-$ Shrink wrap, $\mathrm{P}_{5^{-}}$Control 
Postharvest quality of Rambutan (Nephelium lappaceum L.)

Table 5. Effect of pre-packaging on reducing sugar (\%) of rambutan fruits under different storage conditions

\begin{tabular}{|c|c|c|c|c|c|c|c|c|c|c|c|c|c|c|c|c|c|c|}
\hline \multirow{4}{*}{$\begin{array}{l}\text { Pre- } \\
\text { packaging } \\
\text { Treatment } \\
\text { s }\end{array}$} & \multicolumn{18}{|c|}{ Reducing sugar (\%) } \\
\hline & \multicolumn{18}{|c|}{ Days after storage } \\
\hline & \multicolumn{3}{|c|}{ At storage } & \multicolumn{3}{|c|}{2} & \multicolumn{3}{|c|}{4} & \multicolumn{3}{|c|}{6} & \multicolumn{3}{|c|}{8} & \multirow{2}{*}{$\begin{array}{c}10 \\
(*) S_{2}\end{array}$} & \multirow{2}{*}{$\begin{array}{r}12 \\
S_{2}\end{array}$} & \multirow{2}{*}{$\begin{array}{r}14 \\
S_{2}\end{array}$} \\
\hline & $\mathrm{S}_{1}$ & $\mathrm{~S}_{2}$ & $\begin{array}{l}\text { Mean } \\
\text { (P) }\end{array}$ & $\mathrm{S}_{1}$ & $\mathrm{~S}_{2}$ & $\begin{array}{l}\text { Mea } \\
\text { n (P) }\end{array}$ & $\mathrm{S}_{1}$ & $\mathrm{~S}_{2}$ & $\begin{array}{l}\text { Mea } \\
\text { n (P) }\end{array}$ & $\mathrm{S}_{1}$ & $\mathrm{~S}_{2}$ & $\begin{array}{l}\text { Mea } \\
\text { n (P) }\end{array}$ & $\mathrm{S}_{1}$ & $\mathrm{~S}_{2}$ & $\begin{array}{l}\text { Mea } \\
\mathrm{n}(\mathrm{P})\end{array}$ & & & \\
\hline $\mathrm{P}_{1}$ & 6.96 & 6.96 & 6.96 & 6.40 & 6.80 & 6.60 & 6.53 & 6.96 & 6.66 & 6.04 & 6.73 & 6.38 & 5.15 & 6.41 & 5.78 & 5.93 & 5.50 & 5.14 \\
\hline $\mathrm{P}_{2}$ & 6.80 & 6.80 & 6.80 & 6.53 & 6.96 & 6.75 & 6.61 & 6.80 & 6.78 & 6.58 & 6.80 & 6.69 & 5.24 & 6.41 & 5.83 & 5.98 & 5.55 & 5.22 \\
\hline $\mathrm{P}_{3}$ & 6.59 & 6.59 & 6.59 & 6.14 & 6.59 & 6.37 & 6.11 & 6.59 & 6.35 & 5.87 & 6.59 & 6.08 & 5.02 & 6.27 & 5.69 & 5.75 & 5.21 & 4.89 \\
\hline $\mathrm{P}_{4}$ & 6.96 & 6.96 & 6.96 & 6.33 & 6.96 & 6.65 & 6.47 & 6.96 & 6.71 & 5.90 & 6.80 & 6.35 & 5.29 & 6.37 & 5.83 & 5.87 & 5.39 & 4.96 \\
\hline $\mathrm{P}_{5}$ & 6.59 & 6.59 & 6.59 & 6.17 & 6.59 & 6.38 & 5.98 & 6.59 & 6.28 & 5.06 & 6.30 & 5.68 & 4.09 & 5.89 & 4.99 & 4.83 & 4.16 & 3.59 \\
\hline Mean (S) & 6.78 & 6.78 & & 6.31 & 6.78 & & 6.34 & 6.78 & & 5.68 & 6.64 & & 4.96 & 6.25 & & 5.67 & 5.16 & 4.76 \\
\hline $\mathrm{CD}(0.05)$ & $\begin{array}{l}\text { P- NS } \\
\text { S- NS } \\
\text { PxS- }\end{array}$ & & & $\begin{array}{l}\text { P-NS } \\
\text { S-NS } \\
\text { PxS- }\end{array}$ & & & $\begin{array}{l}\text { P-0.5 } \\
\text { S-0.3 } \\
\text { PxS- }\end{array}$ & & & $\begin{array}{l}\text { P-0.4 } \\
\text { S-0.2 } \\
\text { PxS- }\end{array}$ & & & $\begin{array}{l}\text { P-0.3 } \\
\text { S-0.2 } \\
\text { PxS- }\end{array}$ & & & 0.413 & 0.459 & 0.401 \\
\hline
\end{tabular}

$\mathrm{S}_{1}$ - Room temperature $\quad \mathrm{S}_{2}$ - Refrigerated storage

* - Rambutan fruits stored under room temperature $\left(S_{1}\right)$ were discarded due to spoilage after $8^{\text {th }}$ day of storage

$\mathrm{P}_{1}$ - Ventilated LDPE, $\mathrm{P}_{2}$-Ventilated $\mathrm{PP}, \mathrm{P}_{3^{-}}$Polystyrene tray with cling film wrap, $\mathrm{P}_{4}-$ Shrink wrap, $\mathrm{P}_{5^{-}}$Control 
Shetty et al.

Table 6. Effect of pre-packaging on non reducing sugar $(\%)$ of rambutan fruits under different storage conditions

\begin{tabular}{|c|c|c|c|c|c|c|c|c|c|c|c|c|c|c|c|c|c|c|}
\hline \multirow{4}{*}{$\begin{array}{l}\text { Pre } \\
- \\
\text { pac } \\
\text { kag } \\
\text { ing } \\
\text { Tre } \\
\text { atm } \\
\text { ent } \\
\text { s }\end{array}$} & \multicolumn{18}{|c|}{ Non reducing sugar $(\%)$} \\
\hline & \multicolumn{18}{|c|}{ Days after storage } \\
\hline & \multicolumn{3}{|c|}{ At storage } & \multicolumn{3}{|c|}{2} & \multicolumn{3}{|c|}{4} & \multicolumn{3}{|c|}{6} & \multicolumn{3}{|c|}{8} & \multirow{2}{*}{$\begin{array}{l}10 \\
(*) \\
S_{2}\end{array}$} & \multirow{2}{*}{\begin{tabular}{|l}
12 \\
$S_{2}$
\end{tabular}} & \multirow{2}{*}{$\frac{14}{S_{2}}$} \\
\hline & $\mathrm{S}_{1}$ & $\mathrm{~S}_{2}$ & $\begin{array}{l}\text { Mean } \\
\text { (P) }\end{array}$ & $\mathrm{S}_{1}$ & $\mathrm{~S}_{2}$ & $\begin{array}{l}\text { Mean } \\
(\mathrm{P})\end{array}$ & $\mathrm{S}_{1}$ & $\mathrm{~S}_{2}$ & $\begin{array}{l}\text { Mean } \\
\text { (P) }\end{array}$ & $\mathrm{S}_{1}$ & $\mathrm{~S}_{2}$ & $\begin{array}{l}\text { Mean } \\
(\mathrm{P})\end{array}$ & $\mathrm{S}_{1}$ & $\mathrm{~S}_{2}$ & $\begin{array}{l}\text { Mean } \\
(\mathrm{P})\end{array}$ & & & \\
\hline $\mathrm{P}_{1}$ & 11.68 & 11.68 & 11.68 & 11.84 & 11.59 & 11.71 & 12.17 & 11.52 & 11.84 & 10.60 & 10.43 & 10.51 & 10.40 & 10.56 & 10.48 & 10.44 & 9.87 & 9.08 \\
\hline $\mathrm{P}_{2}$ & 12.34 & 12.34 & 12.34 & 12.08 & 12.22 & 12.15 & 11.70 & 12.14 & 11.92 & 10.77 & 10.73 & 10.75 & 10.45 & 10.91 & 10.68 & 10.60 & 10.06 & 9.22 \\
\hline $\mathrm{P}_{3}$ & 12.61 & 12.61 & 12.61 & 12.39 & 12.34 & 12.37 & 12.19 & 12.18 & 12.18 & 11.07 & 10.73 & 10.90 & 10.49 & 10.64 & 10.56 & 10.49 & 9.81 & 9.22 \\
\hline $\mathrm{P}_{4}$ & 12.30 & 12.30 & 12.30 & 12.56 & 12.11 & 12.34 & 12.20 & 11.98 & 12.09 & 10.98 & 10.69 & 10.83 & 10.16 & 10.74 & 10.45 & 10.40 & 9.72 & 9.17 \\
\hline $\mathrm{P}_{5}$ & 12.06 & 12.06 & 12.06 & 10.95 & 12.01 & 11.48 & 10.89 & 12.01 & 11.45 & 9.59 & 10.30 & 9.94 & 8.37 & 9.69 & 9.03 & 8.51 & 7.92 & 7.15 \\
\hline $\begin{array}{l}\mathrm{Me} \\
\text { an } \\
(\mathrm{S})\end{array}$ & 12.20 & 12.20 & & 11.67 & 12.06 & & 11.92 & 11.97 & & 10.60 & 10.57 & & 9.97 & 10.51 & & 10.08 & 9.47 & 8.76 \\
\hline $\begin{array}{l}\mathrm{CD} \\
(0.0 \\
5)\end{array}$ & $\begin{array}{l}\text { P- NS } \\
\text { S- NS } \\
\text { PxS- }\end{array}$ & & & $\begin{array}{l}\text { P-0.75 } \\
\text { S-0.47 } \\
\text { PxS-1. }\end{array}$ & & & $\begin{array}{l}\text { P-0.80 } \\
\text { S-0.51 } \\
\text { PxS-1 }\end{array}$ & & & $\begin{array}{l}\text { P-0.78 } \\
\text { S-0.49 } \\
\text { PxS-1 }\end{array}$ & & & $\begin{array}{l}\text { P-0.63 } \\
\text { S-0.40 } \\
\text { PxS-0 }\end{array}$ & & & 0.413 & 0.559 & 0.601 \\
\hline
\end{tabular}

$\mathrm{S}_{1}$ - Room temperature $\quad \mathrm{S}_{2}$ - Refrigerated storage

* - Rambutan fruits stored under room temperature $\left(S_{1}\right)$ were discarded due to spoilage after $8^{\text {th }}$ day of storage

$\mathrm{P}_{1}$ - Ventilated LDPE, $\mathrm{P}_{2}$-Ventilated $\mathrm{PP}, \mathrm{P}_{3^{-}}$Polystyrene tray with cling film wrap, $\mathrm{P}_{4}-$ Shrink wrap, $\mathrm{P}_{5^{-}}$Control 
Postharvest quality of Rambutan (Nephelium lappaceum L.)

Table 7. Effect of pre-packaging on antioxidant activity $(\%)$ of rambutan fruits under different storage conditions

\begin{tabular}{|c|c|c|c|c|c|c|c|c|c|c|c|c|c|c|c|c|c|c|}
\hline \multirow{4}{*}{$\begin{array}{l}\text { Pre } \\
- \\
\text { pac } \\
\text { kag } \\
\text { ing } \\
\text { Tre } \\
\text { atm } \\
\text { ent } \\
\text { s }\end{array}$} & \multicolumn{18}{|c|}{ Antioxidant activity (\%) } \\
\hline & \multicolumn{18}{|c|}{ Days after storage } \\
\hline & \multicolumn{3}{|c|}{ At storage } & \multicolumn{3}{|c|}{2} & \multicolumn{3}{|c|}{4} & \multicolumn{3}{|c|}{6} & \multicolumn{3}{|c|}{8} & \multirow{2}{*}{$\begin{array}{l}10 \\
(*) \\
S_{2}\end{array}$} & \multirow{2}{*}{$\begin{array}{r}12 \\
S_{2}\end{array}$} & \multirow{2}{*}{$\begin{array}{r}14 \\
S_{2}\end{array}$} \\
\hline & $\mathrm{S}_{1}$ & $\mathrm{~S}_{2}$ & $\begin{array}{l}\text { Mean } \\
(\mathrm{P})\end{array}$ & $\mathrm{S}_{1}$ & $\mathrm{~S}_{2}$ & $\begin{array}{l}\text { Mean } \\
(\mathrm{P})\end{array}$ & $\mathrm{S}_{1}$ & $\mathrm{~S}_{2}$ & $\begin{array}{l}\text { Mean } \\
(\mathrm{P})\end{array}$ & $\mathrm{S}_{1}$ & $\mathrm{~S}_{2}$ & $\begin{array}{l}\text { Mean } \\
(\mathrm{P})\end{array}$ & $\mathrm{S}_{1}$ & $\mathrm{~S}_{2}$ & $\begin{array}{l}\text { Mean } \\
\text { (P) }\end{array}$ & & & \\
\hline $\mathrm{P}_{1}$ & 88.15 & 88.15 & 88.15 & 82.73 & 85.84 & 84.18 & 79.48 & 82.73 & 81.10 & 73.03 & 79.48 & 76.26 & 64.72 & 74.92 & 69.82 & 70.77 & 67.62 & 61.89 \\
\hline $\mathrm{P}_{2}$ & 88.09 & 88.09 & 88.09 & 83.88 & 86.57 & 85.22 & 79.96 & 83.88 & 81.92 & 74.04 & 80.29 & 77.17 & 66.34 & 76.66 & 71.50 & 72.30 & 69.13 & 64.23 \\
\hline $\mathrm{P}_{3}$ & 88.29 & 88.29 & 88.29 & 81.00 & 84.10 & 82.55 & 75.85 & 81.00 & 78.42 & 70.20 & 75.85 & 73.02 & 63.88 & 73.23 & 68.54 & 69.85 & 64.45 & 59.69 \\
\hline $\mathrm{P}_{4}$ & 88.11 & 88.11 & 88.11 & 80.47 & 85.64 & 83.15 & 76.67 & 80.47 & 78.57 & 69.85 & 76.67 & 73.26 & 62.59 & 74.29 & 68.44 & 69.87 & 62.59 & 56.73 \\
\hline $\mathrm{P}_{5}$ & 88.50 & 88.50 & 88.50 & 79.04 & 83.86 & 81.45 & 71.25 & 79.04 & 75.15 & 64.02 & 71.25 & 67.64 & 55.12 & 64.21 & 59.67 & 59.69 & 53.76 & 46.57 \\
\hline $\begin{array}{l}\mathrm{Me} \\
\text { an } \\
(\mathrm{S}) \\
\end{array}$ & 88.03 & 88.03 & & 81.42 & 85.20 & & 76.64 & 81.42 & & 70.23 & 76.71 & & 62.53 & 72.66 & & 68.49 & 63.51 & $\begin{array}{l}57.82 \\
2\end{array}$ \\
\hline
\end{tabular}

$\mathrm{S}_{1}$ - Room temperature $\quad \mathrm{S}_{2}$ - Refrigerated storage

* - Rambutan fruits stored under room temperature $\left(S_{1}\right)$ were discarded due to spoilage after $8^{\text {th }}$ day of storage

$\mathrm{P}_{1}$ - LDPE, $\mathrm{P}_{2}-\mathrm{PP}, \mathrm{P}_{3}$ - Polystyrene tray with cling film wrap, $\mathrm{P}_{4}-$ Shrink wrap, $\mathrm{P}_{5}-$ Control 
reducing sugar of $4.99 \%$. Pre-packaging treatments $\left(\mathrm{P}_{1} \mathrm{~S}_{2}, \mathrm{P}_{2} \mathrm{~S}_{2}, \mathrm{P}_{3} \mathrm{~S}_{2}, \mathrm{P}_{4} \mathrm{~S}_{2}\right)$ noticed no significant difference and ranged from $6.27 \%$ to $6.41 \%$ and lowest reducing sugar was recorded in $\mathrm{P}_{5} \mathrm{~S}_{2}$ (control + refrigerated storage) as $5.89 \%$ after 8 days of storage under refrigerated condition similar fashion was observed in room temperature with insignificant difference among pre-packaging treatments $\left(\mathrm{P}_{1} \mathrm{~S}_{1}, \mathrm{P}_{2} \mathrm{~S}_{1}, \mathrm{P}_{3} \mathrm{~S}_{1}, \mathrm{P}_{4} \mathrm{~S}_{1}\right)$ and lowest reducing sugar content was recorded by $\mathrm{P}_{5} \mathrm{~S}_{1}$ (control + room temperature) as $4.09 \%$. Prepackaged fruits stored under room temperature were discarded due to spoilage after $8^{\text {th }}$ day of storage. After $14^{\text {th }}$ day of storage, rambutan fruits showed no significant difference in reducing sugar for the pre-packaging treatments $\left(\mathrm{P}_{1} \mathrm{~S}_{2}, \mathrm{P}_{2} \mathrm{~S}_{2}, \mathrm{P}_{3} \mathrm{~S}_{2}\right.$, $\left.\mathrm{P}_{4} \mathrm{~S}_{2}\right)$ at refrigerated storage and ranged from $4.89 \%$ to $5.22 \%$ while the lowest reducing sugar was recorded by $\mathrm{P}_{5} \mathrm{~S}_{2}$ (control + refrigerated storage) as $3.59 \%$ (Table 5).Non-reducing sugar of fruits stored at room temperature was $10.60 \%$ and $10.57 \%$ at refrigerated condition with no significant difference after 6 days of storage whereas it was $9.97 \%$ and $10.51 \%$ for fruits stored under room temperature and refrigerated storage respectively after 8 days of storage. All the pre-packaging treatments showed no significant difference between themselves and lowest non-reducing sugar content was recorded in $\mathrm{P}_{5}$ (without pre-packaging) as $9.03 \%$. When the interaction effects were studied, all the pre-packaging treatments $\left(\mathrm{P}_{2} \mathrm{~S}_{2}\right.$, $\mathrm{P}_{4} \mathrm{~S}_{2}, \quad \mathrm{P}_{3} \mathrm{~S}_{2}$ and $\left.\mathrm{P}_{1} \mathrm{~S}_{2}\right)$ showed no significant difference. Lowest non-reducing sugar was noticed in $\mathrm{P}_{5} \mathrm{~S}_{2}$ (control + refrigerated storage) $(9.69 \%)$ after 8 days of storage under refrigerated condition. At room temperature $\mathrm{P}_{1} \mathrm{~S}_{1}, \mathrm{P}_{2} \mathrm{~S}_{1}, \mathrm{P}_{3} \mathrm{~S}_{1}$ and $\mathrm{P}_{4} \mathrm{~S}_{1}$ showed no difference. The lowest non-reducing sugar of $8.37 \%$ was for $\mathrm{P}_{5} \mathrm{~S}_{1}$ (control + room temperature). Pre-packaged rambutan fruits stored under room temperature were discarded due to spoilage after $8^{\text {th }}$ day of storage. After $14^{\text {th }}$ day of storage, rambutan fruits showed no significant difference in non-reducing sugar for the prepackaging treatments $\left(\mathrm{P}_{1} \mathrm{~S}_{2}, \mathrm{P}_{2} \mathrm{~S}_{2}, \mathrm{P}_{3} \mathrm{~S}_{2}, \mathrm{P}_{4} \mathrm{~S}_{2}\right)$ at refrigerated storage and ranged from $9.08 \%$ to $9.22 \%$. The lowest non-reducing sugar content was recorded in treatment $\mathrm{P}_{5} \mathrm{~S}_{2}$ (control + refrigerated storage) after $12^{\text {th }}$ and $14^{\text {th }}$ days of storage as $7.92 \%$ and $7.15 \%$ respectively (Table 6). Reducing and non-reducing sugar of rambutan fruits decreased with storage and reduction was highest in room temperature than refrigerated storage. Fruits without packaging recorded lowest reducing and non-reducing sugar during the whole storage period whereas all pre-packaging treatments helped in better retention. This result had synchronization with Tefera et al. (2007) in mango. Higher reduction in non-reducing sugar stored at room temperature as compared to refrigerated storage might be due to the low temperature and relative humidity compared to the ambient storage (Seyoum, 2002).Total sugar of fruits stored at room temperature was recorded as $16.29 \%$ and $14.87 \%$ respectively after 6 and 8 days of storage whereas it was $17.22 \%$ and $16.76 \%$ respectively under refrigerated storage. Among the pre-packaging treatments, highest total sugar of $17.18 \%$ and $16.51 \%$ was recorded for $\mathrm{P}_{2}$ (ventilated PP) after $6^{\text {th }}$ and $8^{\text {th }}$ day of storage respectively which did not differ significantly with shrink wrapping $\left(\mathrm{P}_{4}\right)$ for both storage days and the lowest was recorded by $\mathrm{P}_{5}$ (without pre-packaging) as $15.62 \%$ and $14.02 \%$ after 6 and 8 days of storage respectively. When the interaction effects were studied, after $8^{\text {th }}$ day of storage also no significant difference was noticed among the pre-packaging treatments $\mathrm{P}_{1} \mathrm{~S}_{2}, \mathrm{P}_{2} \mathrm{~S}_{2}$, $\mathrm{P}_{3} \mathrm{~S}_{2}$ and $\mathrm{P}_{4} \mathrm{~S}_{2}$ under refrigerated storage. $\mathrm{P}_{2} \mathrm{~S}_{1}$ (ventilated $\mathrm{PP}+$ room temperature) showed highest retention of total sugar (15.70\%) and showed no difference with $\mathrm{P}_{4} \mathrm{~S}_{1}$ (shrink wrapping + room temperature) under room temperature. Fruits without pre-packaging (control) recorded the lowest total sugar of $15.58 \%$ and $12.47 \%$ under refrigerated and room temperature storage respectively. After $14^{\text {th }}$ day of storage, ventilated $\mathrm{PP}+$ refrigerated storage $\left(\mathrm{P}_{2} \mathrm{~S}_{2}\right)$ recorded the highest total sugar of $15.45 \%$ followed by $\mathrm{P}_{4} \mathrm{~S}_{2}$ (shrink wrapping+ refrigerated storage) $14.22 \%$ (Table 4). The lowest total sugar of $13.19 \%$ and $11.74 \%$ was recorded in $\mathrm{P}_{5} \mathrm{~S}_{2}$ (control + refrigerated storage) after $12^{\text {th }}$ and $14^{\text {th }}$ day of storage respectively. Total sugar content of rambutan fruits ranged between $19.14 \%$ and $19.27 \%$ at the time of storage. Rambutan stored under refrigerated condition retained the highest total sugar $(15.45 \%)$ even after 14 days of storage and the fruits stored at room temperature recorded highest reduction in total sugar content. The decrease in total sugar at room temperature could be due to higher rates of metabolic activity and respiration resulting in quick 
Postharvest quality of Rambutan (Nephelium lappaceum $\mathbf{L}$.)
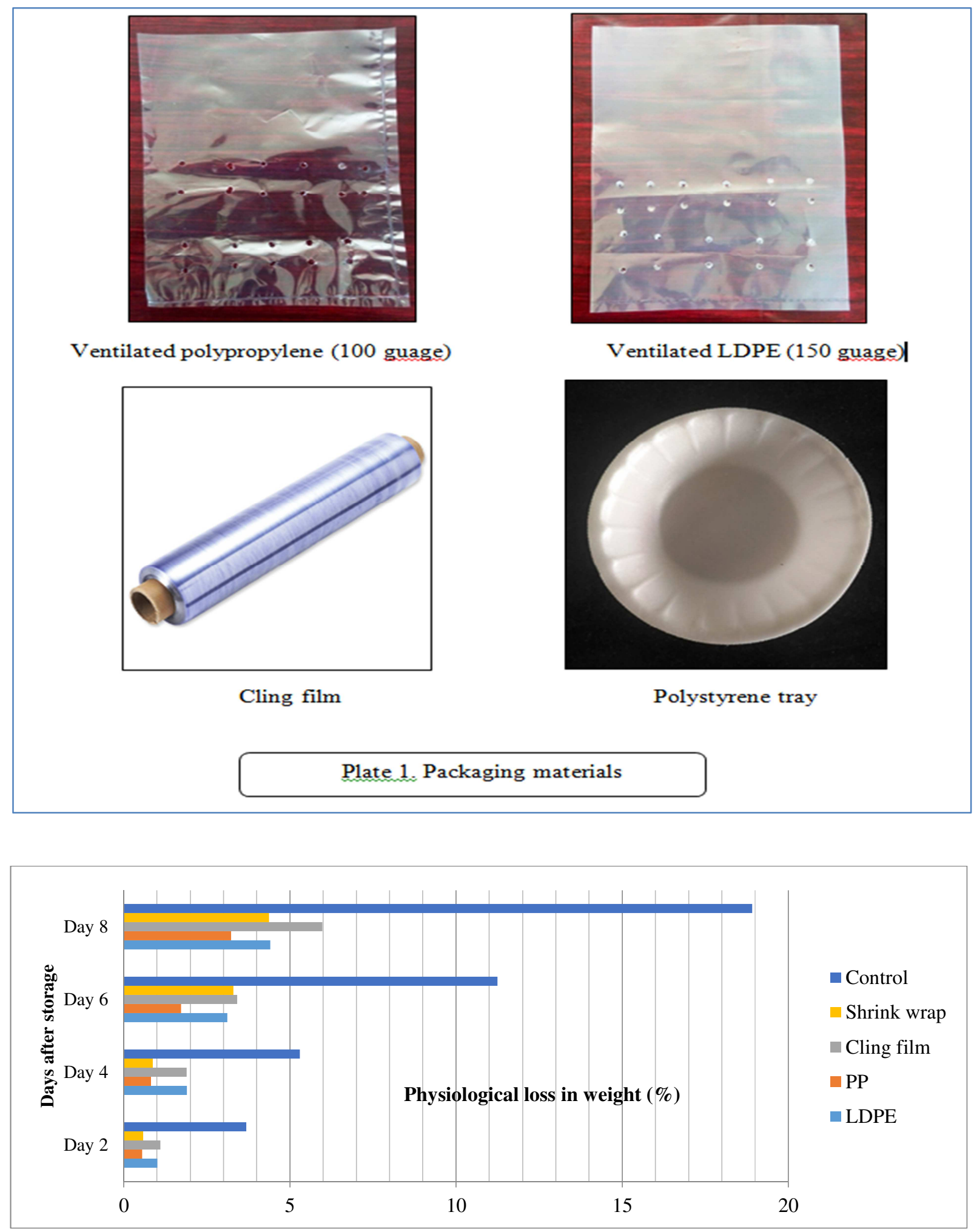

Fig 1a. Effect of pre-packaging on PLW (\%) of rambutan fruits under room temperature

Environment Conservation Journal 


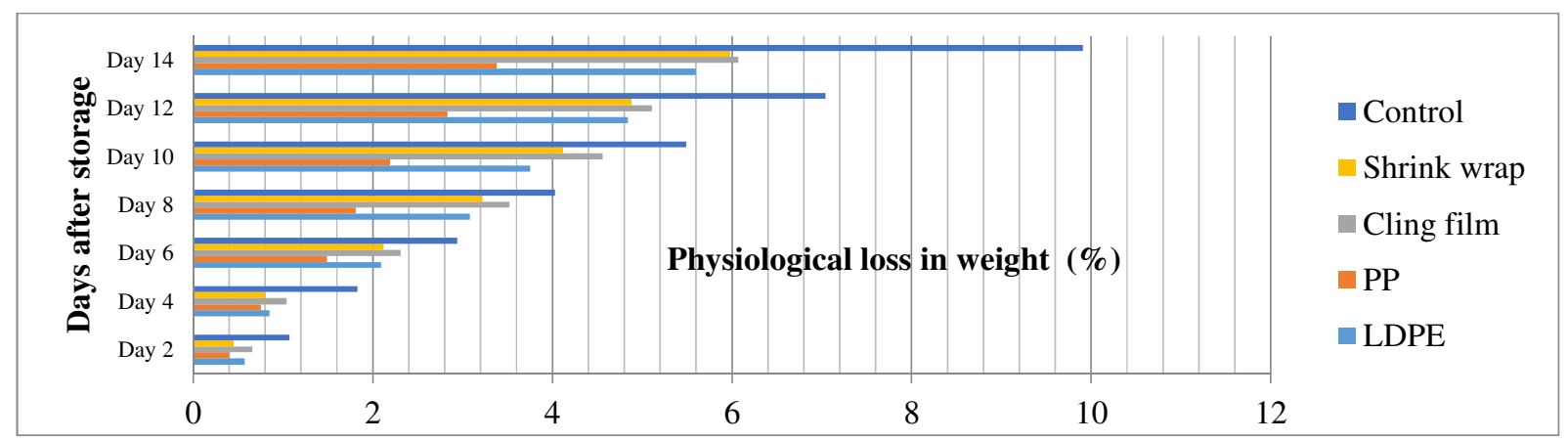

Fig 1b. Effect of pre-packaging PLW (\%) of rambutan fruits under refrigerated storage

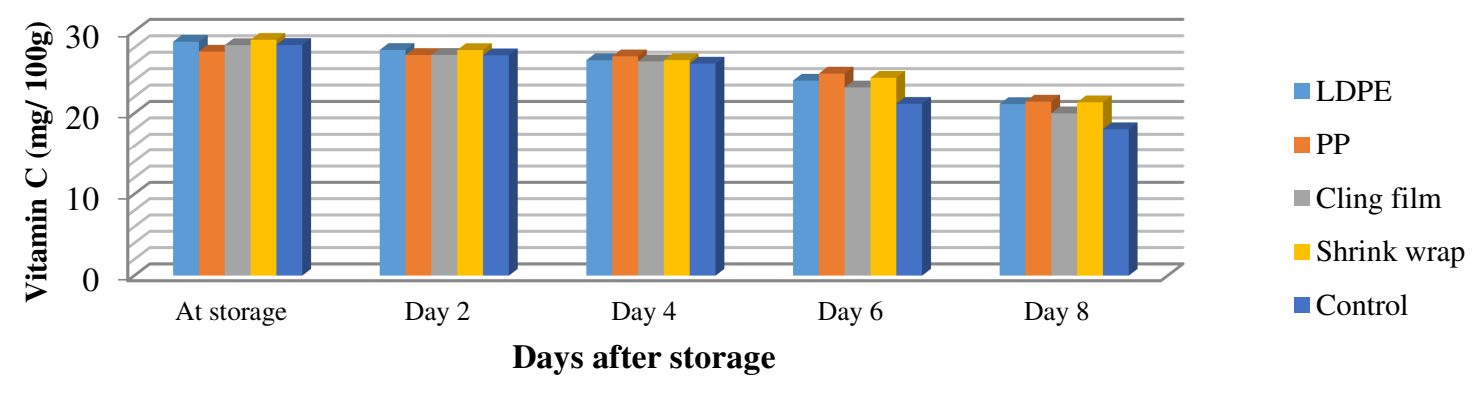

Fig 2a. Effect of pre-packaging on vitamin C (mg/ 100g) of rambutan fruits under room temperature

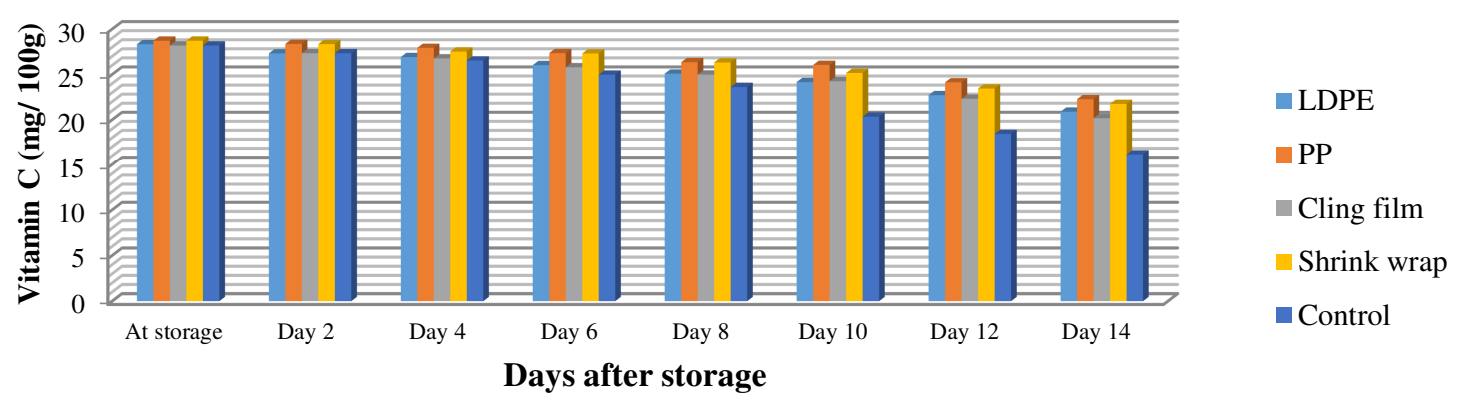

Fig 2b. Effect of pre-packaging on vitamin C (mg/ 100g) of rambutan fruits under refrigerated storage

hydrolysis of sugar (Gomez et al., 2002). Pre- refrigerated storage. Among the pre-packaging packaging of rambutan in ventilated PP stored at treatments, $\mathrm{P}_{2}$ (ventilated PP) had (ventilated PP) room temperature retained maximum total sugar of had highest antioxidant activity of $81.92 \%, 77.17 \%$ $15.70 \%$ after $8^{\text {th }}$ day of storage. When fruits were and $71.50 \%$ followed by $\mathrm{P}_{1}$ (ventilated LDPE) with packed with pre-packaging materials it created $81.10 \%, 76.26 \%, 69.82 \%$ after $4^{\text {th }}, 6^{\text {th }}$ and $8^{\text {th }}$ days modified atmosphere where consumption of of storage and the lowest value was noticed in $\mathrm{P}_{5}$ respiratory substrates like sugar are retarded (without pre-packaging) as 75.15\%, 67.64\% and (Chachin et al., 2002). Antioxidant activity of fruits $59.67 \%$ respectively. When the interaction effects recorded $70.23 \%$ at room temperature after 6 days were studied, after $8^{\text {th }}$ day of storage, treatment $\mathrm{P}_{2} \mathrm{~S}_{2}$ of storage and $62.53 \%$ after 8 days of storage while (ventilated PP + refrigerated storage) had the it was $76.71 \%$ and $72.66 \%$ respectively under highest antioxidant activity of $76.66 \%$ followed by 
$\mathrm{P}_{1} \mathrm{~S}_{2}$ (ventilated LDPE + refrigerated storage) $(64.72 \%)$ and the treatment $\mathrm{P}_{2} \mathrm{~S}_{1}$ (ventilated $\mathrm{PP}+$ room temperature) recorded the antioxidant activity of $66.34 \%$ followed by $\mathrm{P}_{1} \mathrm{~S}_{1}$ (ventilated LDPE + room temperature) $(64.72 \%)$ for room temperature storage. The lowest antioxidant activity of $64.21 \%$ was for $\mathrm{P}_{5} \mathrm{~S}_{2}$ (control + refrigerated storage) and $55.12 \%$ for $\mathrm{P}_{5} \mathrm{~S}_{1}$ (control + room temperature). Rambutan fruits stored under room temperature were discarded due to spoilage after $8^{\text {th }}$ day of storage. The treatment $\mathrm{P}_{2} \mathrm{~S}_{2}$ (ventilated $\mathrm{PP}+$ refrigerated storage) recorded the highest antioxidant activity of $69.13 \%$ and $64.23 \%$ after $12^{\text {th }}$ and $14^{\text {th }}$ day of storage respectively. The lowest antioxidant activity was recorded in treatment $\mathrm{P}_{5} \mathrm{~S}_{2}$ (control + refrigerated storage) after $12^{\text {th }}$ and $14^{\text {th }}$ days of storage as $53.76 \%$ and $46.57 \%$ respectively (Table 7). Reduction of antioxidant activity of fruits in storage was attributed to the reduction in total phenol content and vitamin C.

On analyzing PLW, and biochemical parameters ventilated PP was found acceptable up to 8 days at room temperature and 14 days at refrigerated condition while untreated fruits had shelf life of only five days under both the storage conditions. The fruits stored without any pre-packaging recorded shelf life of 5 days at room temperature and refrigerated condition whereas fruits prepackaged in ventilated PP stored under room temperature exhibited a shelf life of 8 days and 14 days under refrigerated condition. This result is in accordance with Widjanarko et al. (2000) in rambutan fruits packaged with polypropylene at low temperature $\left(10 \pm 1{ }^{\circ} \mathrm{C}\right)$ extended shelf life up to 12 days. The extension of shelf life at low temperature was due to reduction in metabolism of fruits (Latifah et al., 2009; Julianti et al., 2012; Shao et al., 2013). Intensity of spoilage of prepackaged rambutan fruits stored at room temperature was high as compared to refrigerated condition. Thus shelf life of rambutan fruit was influenced by pre-packaging treatments and storage temperature.

\section{Conclusion}

Pre-packaging as well as storage temperature influenced the biochemical parameters of rambutan during storage. Loss of biochemical parameters was greater with increase in storage temperature and duration. Under refrigerated storage, ventilated PP was ideal as it registered the lowest PLW and excellent biochemical parameters after 14 days of storage. Rambutan fruits without any postharvest treatments had limited shelf life of only 3 days with diminished nutritional and organoleptic qualities. Pre-packaging in ventilated polypropylene (100 guage) could extend the shelf life of fruits up to fourteen days when stored under refrigerated condition and eight days under room temperature storage with minimum nutritional loss.

\section{References}

Ahvenainen, R., 1996. New approaches in improving the shelf life of minimally processed fruits and vegetables. Trends Food Sci. Technol. 7: 179-187.

Assumi, S.R., Piloo, N. and Kabir, J., 2009. Effect of plastic films on shelf life of okra. Andhra Agric. J. 56(1): 102106.

Azene, M., Seyoum, W. and Kebede, W., 2014. Effect of packaging materials and storage environment on postharvest quality of papaya fruit. J. Food Sci. Technol. 51 (6): 1041-1055.

Chachin, K., Unda, Y., Imahori, Y. and Wang, C.Y., 2002. The effects of modified atmosphere packaging on the storage life of loquat fruits. Postharvest Biol. Technol. 24: 341348.

Desai, U.T., Kadam, S.S. and Saliunkhe, D.K., 1986. Postharvest handling, storage and processing of vegetables. In: Bose, T. K. and Som, M. S. (ed) Naya Prokash, Bidhan Sharani, Kolkata, India, 206p.

Gast, K.L.B., 1991. Containers and packaging of fruits and vegetables. Kansas State University [online]. Available: http://www.oznet.ksu.edu [06 febraury 2017].

Getenit, H., Workneh, T.S. and Woldetsdik, K., 2008. The effect of cultivar, maturity stage and storage environment on quality of tomatoes. J. Food. Engg. 87: 467-498.

Gomez, M., Lajolo, F. and Cordenunsi, B., 2002. Evolution of soluble sugars during ripening of fruit and its relation to sweet taste. J. Food Sci. 67: 442-447.

Julianti, E., Ridwansyah., Yusraini, E. and Suhaidi, I., 2012. Effect of modified atmosphere packaging on postharvest quality of Rambutan $c v$. Binjai. J. Food Sci. engg. 2: 111117.

Kays, S.J. and Paull, R.E. 2004. Stress in harvested products. In: Postharvest biology. Exon press, Athens, GA. 35-414.

Lam, P.F., Kosiyachinda, S., Lizada, M.C.C., Mendonza, D.B., Prabawati, S. and Lee, S.K. 1987. Postharvest physiology 


\section{Shetty et al.}

and storage of rambutan. In: Lam, P. F and Kosiyachinda, S. (eds), Rambutan Fruit Development, Postharvest Physiology and Marketing in ASEAN. ASEAN Food Handling Bureau, Kaula Lumpur, pp. 39-50.

Latifah, M.N., Abdulalh, H., Ab-Aziz, I., Fauziah, O. and Talib, Y. 2009. Quality changes of rambutan fruit in different packaging system. J. Trop. Agric. Food Sci. 37(2). 143-151

Mendonza, D.B., Pantastico, E.B. and Javier, F.B., 1972. Storage and handling of rambutan. Phillipp. Agric. 55: 322-332.

Mitchell, F.G., 1991. 'Cooling horticultural commodities. In: Kader, A. A. Postharvest technology of horticultural crops'. University of California, Publication. 3311(2): 5368.

Nainar, P., Balakrishnan, K., Pappaiah, C.M. and Sambandamurthi, S., 1997. Studies on improving the shelf life of green amaranthus through different storage methods. S. Ind. Hortic. 45(5): 293-296.

O'Hare, T.J., 1995. Postharvest physiology and storage of rambutan. Postharvest Biol.Technol. 6: 189-199.

O'Hare, T.J., Prasad, A. and Cooke, A.W. 1994. Low temperature and controlled atmosphere storage of rambutan. Postharvest Biol. Technol. 4: 147-15.

Onyango, C.M. 2010., Preharvest and postharvest factors affecting yield and nutrient contents of vegetable amaranth (var. Amaranthus hypochondrichus). Ph.D. thesis, Wageningen University, Wageningen, 132p.

Paine, F.A. and Paine, H.Y., 1992. A Handbook on Food Packaging. $2^{\text {nd }}$ Ed. Balckie Academic and Professional, Glasgow.

Pantastico Er, B., (Editor)., 1975. Postharvest physiology, Handling and Utilization of Tropical and Sub Tropical Fruits and Vegetables. AVI Publishing, Westport, CT. 120$188 \mathrm{pp}$.
Pantastico Er, B., 1995. Postharvest physiology, handling and utilization of tropical and sub-tropical fruits and vegetables. AVI Publishers. WestPort, Connecticut:560.

Ranganna, S., 1986. Handbook of Analysis and quality control for fruit and vegetable products. Tata McGraw Hill Publishing Company Limited. New Delhi:182.

Sadasivam, S. and Manickam, A., 1992. Biochemical method for agricultural sciences. Wiley Eastern Limited and Tamil Nadu Agricultural University, Coimbatore: 246.

Seyoum, T.M., 2002. The improvement of shelf life of vegetables through the pre and post-harvest treatment. Trop. Agric. J. 81(3): 179-185.

Shao, Y., Xie, J., Chen, P. and Li, W. 2013. Changes in some chemical components and in the physiology of rambutan fruit (Nephelium lappaceum L.) as affected by storage temperature and packing material. Fruits. 68: 15-24.

Sharma, R. and Bhat, T. K., 2009. DPPH antioxidant assay revisited. Food Chem. 113: 1202-1205.

Singh, S.P. and Rao, D.V., 2005. Effect of modified atmosphere packaging on allevation of chilling injury and dietary antioxidant levels in solo papaya during low temperature storage. Europe J. Hort. Sci. 70: 246-252.

Tefera, A., Workneh, T.S. and Woldestadik, K., 2007. Effect of disinfection, packaging and storage environment on shelf life of mango. Bios Engg. 96: 1537-1550.

Widjanarko, S.B., Trisnawati, C.H.Y. and Susanto, T., 2000. Changes in respiration, composition and sensory characteristics of rambutan packed with plastic films during storage at low temperature. J. Agric. Technol. 1(3): $1-8$.

Wills, R.B.H., Lee, T.H., Graham. D., Mc Glasson, W.B. and Hall, E.G., 1989. Post-harvest: An introduction to the physiological and handling of Fruits and Vegetables. $\mathrm{CAB}$ international, Wallingford, UK. 5: 250. 